\title{
Laparoscopic Appendicectomy in a 26-Weeks Parturient
}

\author{
Reshama Manoharrao Kailuke ${ }^{1}$ Bhavitha Venigalla², Sanjot Sudhir Ninave ${ }^{3}$
}

\begin{abstract}
${ }^{1}$ Department of Anaesthesiology, Datta Meghe Institute of Medial Sciences, Jawaharlal Nehru Medical College, Sawangi, Wardha, Maharashtra, India. ${ }^{2}$ Department of Anaesthesiology, Datta Meghe Institute of Medial Sciences, Jawaharlal Nehru Medical College, Sawangi, Wardha, Maharashtra, India. ${ }^{3}$ Department of Anaesthesiology, Datta Meghe Institute of Medial Sciences, Jawaharlal Nehru Medical College, Sawangi, Wardha, Maharashtra, India.
\end{abstract}

\section{INTRODUCTION}

One of the commonest non-obstetric problem that will require surgical intervention during pregnancy is acute appendicitis. Laparoscopic appendectomy in parturient has many challenges to anesthesiologists like preterm labour, foetal morbidity, and maternal morbidity. Providing safe anaesthesia is such cases is challenging and necessary. The anaesthesiologist has to ensure maintenance of maternal oxygenation, perfusion, and homeostasis, with the least extensive anaesthetic for the best outcome for the foetus.

Laparoscopy in pregnancy is challenging both for the surgeon and anaesthesiologist. Challenges faced by the anaesthesiologist includes laryngoscopy and tracheal intubation associated pressor responses haemodynamic changes associated with gravid uterus causing aortocaval compression and increased in intraabdominal pressures due to pneumoperitoneum, maternal $\mathrm{CO}_{2}$ monitoring to avoid maternal and foetal acidosis, considerations of avoiding foetal exposure to unnecessary medications \& foetal heart rate monitoring. Whenever practically feasible regional anaesthesia is usually preferred over general anaesthesia in pregnancy whenever it is practically feasible. Regional anaesthesia in this case involves discomfort to the patient, need to take a higher level for surgical anaesthesia, hemodynamic changes associated after induction, risk of uterus manipulation by surgeons all of which can predispose to preterm labour. In our case we have opted for general anaesthesia over regional anesthesia due to fore mentioned reasons.

\section{PRESENTATION OF CASE}

A 25-year-old parturient, with 26 weeks of gestation came with complaints of abdominal pain and fever. There was no significant previous surgical/medical history. On examination she had tachycardia $115 / \mathrm{min}$ and a temperature of $38.3^{\circ} \mathrm{C}$, with right lower quadrant pain, all routine laboratory investigations (complete blood count, liver and kidney profile tests coagulation profile) were done. There was mild leukocytosis 11000 /cumm with others within normal limits. Radiological evaluation in addition to clinical picture reveled acute appendicitis. Patient was taken for emergency laparoscopic appendectomy. Preanaesthetic evaluation was done; an informed and written consent was obtained from patient and her husband. NBM of 6 hours was confirmed. Preoperatively Inj. Metoclopramide was given 2 hours before the surgery. Preoperative foetal wellbeing was assessed and documented by the obstetrician. Preoperatively injection Isoxsuprine $10 \mathrm{mg}$ is started in D5.
Corresponding Author:

Dr. Bhavitha Venigalla,

Flat No. 9, Meghe Heights-3,

Datta Meghe Institute of Medial Sciences, Sawangi, Meghe-4422001,

Maharashtra, India.

E-mail: bhavitha123@gmail.com

DOI: 10.14260/jemds/2020/427

How to Cite This Article:

Kailuke RM, Venigalla B, Ninave SS Laparoscopic appendcectomy in 26 weeks parturient. J. Evolution Med. Dent. Sci. 2020;9(27):1964-1966, DOI: 10.14260/jemds/2020/427

Submission 15-04-2020,

Peer Review 01-06-2020,

Acceptance 10-06-2020,

Published 06-07-2020.

Copyright (C) 2020 JEMDS. This is an open access article distributed under Creative Commons Attribution License [Attribution 4.0 International (CC BY 4.0)] 
The patient's intraoperative monitoring included electrocardiogram, noninvasive blood pressure, pulse oximetry, capnography, as well as foetal heart rate monitoring, as the latter alerts us early compromise of the foetus. Nonparticulate antacid $0.3 \mathrm{M}$ sodium citrate was given for aspiration prophylaxis. To avoid the aortocaval compression by the gravid uterus wedge is placed under right thigh. Induction of anaesthesia started with administration of injection glycopyrrolate $0.2 \mathrm{mg}$ I.V., rapid sequence induction was performed administering Injection Propofol $100 \mathrm{mg}$ I.V. and succinyl choline $75 \mathrm{mg}$ I.V. using Sellick's manoeuvre. Intubation was done using $7.5 \mathrm{~mm}$ Cuffed Endotracheal tube, bilateral air entry checked and tube secured. Anaesthesia was maintained using oxygen: nitrous oxide (50:50) and sevoflurane $2 \%$. Ryles tube was secured to deflate the stomach. Injection Vecuronium is used as muscle relaxant. Patient is catheterised. Intra-abdominal pressure ensured not to be more than $10 \mathrm{mmHg}$. Injection. Paracetamol $1000 \mathrm{mg} \mathrm{I.V.}$ is given as analgesic. Intraoperative vitals were stable. Reversal is ensured using Injection Neostigmine $2.5 \mathrm{mg}+$ Glycopyrrolate $0.5 \mathrm{mg}$. Patient is extubated after satisfying the extubation criteria and shifted to postoperative care unit and started on $100 \%$ oxygen at the rate of 5 liters/minute using Hudson mask with continuous haemodynamic monitoring, level of consciousness as well as foetal heart rate monitoring is done. Intraoperative period was uneventful.

\section{DISCUSSION}

Non-obstetrics surgeries during pregnancy pose challenges to the anaesthesiologist. Taking care of maternal safety and foetal outcome is equally important.(1)

Multidisciplinary team approach involving anaesthesiologists, obstetricians, surgeons \& perinatologists together will be involved in the decision making before proceeding with surgery. Any Elective surgery should be delayed up to 6 weeks postpartum as this will allow resolution of physiological changes of pregnancy Second trimester is chosen for semi-elective surgery, which cannot be postponed. Any Urgent surgery should not be delayed as it may increase secondary complications and increase the risk to the mother and/or foetus. In advanced stages of pregnancy there is a greater risk of uterine irritability and preterm labour, there is no evidence that any anaesthetic technique, agent or dose influences the risk as it is believed it can happen as a result of direct manipulation of the uterus during surgery or the disease process itself.(1)

Refinements in field of surgery, has led to change in approach to laparoscopic surgeries during pregnancy. Benefits of laparoscopy in pregnancy include, decreased postoperative pain, early return of bowel function, early ambulation, decrease hospital stay, with less uterus manipulation.(2),(3),(4) Laparoscopy in pregnancy is double edged sword, it has demerits like iatrogenic injuries to gravid uterus by trocar, preterm labour, maternal hemodynamic changes which can predispose changes uterine blood flow, increased intrabdominal pressures can lead to foetal asphyxia, Maternal and foetal acidosis due to systemic carbon dioxide absorption.(4),(5),(6)
Regional anaesthesia is preferred over general anaesthesia where ever feasible. As it has advantages where the mother maintains her own airway, decreased drug exposure to the foetus minimizes, emesis free recovery and provides good postoperative analgesia.(7) And also disadvantages like patient discomfort, need to take a higher level for surgical anaesthesia, hemodynamic changes associated after induction, risk of uterus manipulation by surgeons all of which can predispose to preterm labour. There is evidence lacking safoety with regional anaesthesia, general anaesthesia is preferred.(7) Epidural analgesia can be an option for postoperative analgesia in pregnant patients undergoing non-obstetric surgery, as it reduces the need of systemic opioids.

Guidelines of the Society of American Gastrointestinal and Endoscopic Surgeons stated many key points helping in the management laparoscopy surgeries in parturient.(3) Obstetrical consultation is to be taken preoperatively and postoperatively with intraoperative foetal heart monitoring is important as it gives us an idea of early compromise, which allow us in optimising maternal haemodynamic and oxygenation, with prompt interventions like altering the maternal position, fluid therapy, hyperventilation,(1),(8) prophylactic perioperative tocolytic therapy showed no proven benefit, but are indicated and considered in $3^{\text {rd }}$ trimester or until resolution of underlying condition or signs of preterm labour are present,(1) prophylaxis is recommended for deep vein thrombosis. (3)

Challenging aspect is management of a airway in pregnancy due to anatomic and physiologic changes like soft tissue oedema of the upper airway, weight gain, breast enlargement, increased mucosal vascularity with an increased propensity to bleed. Rapid sequence induction and intubation is the method of choice for securing the airway. ${ }^{(9)}$ Pregnancy has an increased risk aspiration is higher due to reduced lower oesophageal sphincter tone, increased gastric emptying time and increased intra-abdominal pressure, prophylaxis is to be given for aspiration with H2-receptor antagonists and nonparticulate antacids from 16 weeks onwards.(1)

Important considerations to be taken include patient position - Left lateral decubitus is ideal, Left lateral tilt (20$30^{\circ}$ ) and placement of wedge $(2),(3)$ intrabdominal pressure to be kept in around $10-15 \mathrm{mmHg}$ to prevent foetal acidosis ${ }^{(3)}$ and capnography monitoring of maternal $\mathrm{CO}_{2}$ and foetal heart rate monitoring.

\section{CONCLUSIONS}

Laparoscopic surgery can be performed safely in pregnancy when adequate precautions are taken and concerns are addressed.(2)

\section{REFERENCES}

[1] Upadya M, Saneesh PJ. Anaesthesia for non-obstetric surgery during pregnancy. Indian J Anaesth 2016;60(4):234-41. 
[2] Rajmohan N, Prakasam H, Simy J. Laparoscopic surgeries during second and third trimesters of pregnancy in a tertiary care centre in South India: anaesthetic implications and long-term effects on children. Indian J Anaesth 2013;57(6):612-5.

[3] Pearl J, Price R, Richardson W, et al. Guidelines for diagnosis, treatment and use of laparoscopy for surgical problems during pregnancy. Surg Endosc 2011;25(11):3479-92.

[4] Moreno-Sanz C, Pascual-Pedreño A, Picazo-Yeste JS, et al. Laparoscopic appendectomy during pregnancy: between personal experiences and scientific evidence. J Am Coll Surg 2007;205(1):37-42.

[5] Cruz AM, Southerland LC, Duke T, et al. Intra-abdominal carbon dioxide insufflation in the pregnant ewe. Uterine blood flow, intra-amniotic pressure and cardiopulmonary effects. Anesthesiology 1996;85(6):1395-402.
[6] Upadhyay A, Stanten S, Kazantsev G, et al. Laparoscopic management of a non-obstetric emergency in the third trimester of pregnancy. Surg Endosc 2007;21(8):1344-8.

[7] Nejdlova M, Johnson T. Anaesthesia for non-obstetric procedures during pregnancy. Contin Educ Anaesthesia Crit Care Pain 2012;12(4):203-6.

[8] Higgins MF, Pollard L, McGuinness SK, et al. Foetal heart rate monitoring in non-obstetric surgery: a systematic review of the evidence. Am J Obstet Gynecol MFM 2019;1(4):100048.

[9] Bajwa SJS, Bajwa SK. Anaesthetic challenges and management during pregnancy: strategies revisited. Anesth Essays Res 2013;7(2):160-7. 